\title{
Conversion of pregnenolone to progesterone by mouse morulae and blastocysts
}

\author{
J. T. Wu and Z. H. Liu* \\ Department of Obstetrics and Gynecology, University of Massachusetts Medical School, Worcester, \\ Massachusetts 01655, USA
}

\begin{abstract}
Summary. When mouse morulae, early blastocysts and implanting blastocysts were cultured with tritiated pregnenolone, tritiated progesterone and its metabolites, $5 \alpha$ pregnan-3,20-dione and 3 $\alpha$-hydroxy-5a-pregnan-20-one, were isolated from the medium. It appears that mouse embryos can make progesterone from pregnenolone and the progesterone is quickly metabolized into various metabolites. These abilities increase with development. It is suggested that the mouse embryo can make progesterone and may regulate its own progesterone level for optimal development.
\end{abstract}

Keywords: mouse; blastocyst; progesterone formation; pregnenolone; progesterone metabolites

\section{Introduction}

The conversion of pregnenolone to progesterone is a critical step in the synthesis of steroid hormones. It requires the activity of $\Delta^{5}-3 \beta$-hydroxysteroid dehydrogenase $\left(\Delta^{5}-3 \beta-H S D\right)$ and $\Delta^{5}$-isomerase. Although the $\Delta^{5}-3 \beta$-HSD activity has been shown by a histochemical method to be present in rat, mouse, rabbit and hamster preimplantation embryos (Dickmann et al., 1976), subsequent studies designed to detect the synthesis or accumulation of progesterone in cultures of mouse embryos have yielded only negative results (Sherman \& Atienza, 1977; Antila et al., 1977; Grube et al., 1978). The reasons for the discrepancies are not clear. However, the possibility does exist that the embryos were capable of progesterone synthesis, but that the progesterone was not detected because the amounts formed were: (a) too low to be detected by the methods used or (b) rapidly decreased to undetectable levels by metabolism to other metabolites.

We have re-examined the question of whether preimplantation mouse embryos can convert pregnenolone into progesterone, and to what extent the newly formed progesterone is metabolized.

\section{Materials and Methods}

Recovery and culture of embryos. Mature CF-1 female mice purchased from Charles River Breeding Laboratories (Wilmington, MA, USA) were caged in animal quarters at $20-23^{\circ} \mathrm{C}$, with lights on from $07: 00$ to 19:00 $\mathrm{h}$ EST. They were superovulated with gonadotrophins and mated (Wu \& Lin, 1982). The morning of finding vaginal plugs was considered Day 1 of pregnancy.

The embryos were recovered at 08:30-10:30 h from the oviducts and the uteri on Day 3 and from the uteri on Days 4 and 5. The medium used for flushing and culture was Ham's F-10 modified for in-vitro fertilization procedures (Lopata et al., 1980) and supplemented with bovine serum albumin $(5 \mathrm{mg} / \mathrm{ml}$ : 'Pentex', Miles Scientific, Naperville, IL, USA) and gentamicin $(60 \mu \mathrm{g} / \mathrm{ml}$ : Sigma Chemical, St Louis, MO, USA). The embryos were washed in three changes of medium, and cultured in medium containing $5 \mu \mathrm{M}-\left[7-{ }^{3} \mathrm{H}\right]$ pregnenolone (sp. act. $22 \cdot 5 \mathrm{Ci} / \mathrm{mmol}$ : New England Nuclear, Boston, MA, USA) as follows: Day 3, four groups of 203, 270, 270 and 395 morulae in 15, 18, 18 and $26 \mu \mathrm{l}$ medium, respectively; Day 4 , four groups of $100,105,165$ and 180 blastocysts in $27,17,17$ and $18 \mu \mathrm{l}$ medium, respectively; Day 5, three groups of 90, 147 and 147 implanting blastocysts in 32,33 and $33 \mu \mathrm{l}$ medium,

*Present address: Institute of Zoology, Academia Sinica, Haidian, Beijing, China. 
respectively. The amount of medium in the culture was dictated by the number of embryos recovered and by the age of embryos: the younger the embryo, the higher the embryo density (i.e. the number of embryos per unit volume). A control culture without embryos was provided for each experiment. The cultures were gassed with a humidified mixture of $5 \% \mathrm{CO}_{2}: 95 \%$ air, stoppered and maintained at $37 \% \mathrm{C}$ for 3 (for Day-5 embryos) or 4 (for Day-3 and -4 embryos) days.

Sampling of cultures and analysis of steroids. At the start of culture, two $1-\mu l$ samples were taken from each culture: one for counting of total radioactivity and subsequent calculation of $\left[{ }^{3} \mathrm{H}\right]$ pregnenolone concentration; and one for thin-layer chromatography (t.l.c.) separation of various steroid fractions to establish a baseline value. During culture, a 1- $\mu$ l sample was taken from each culture at $22,46,70$ and $94 \mathrm{~h}$ for steroid analysis. At the end of culture, the whole contents of two cultures from the Day-5 embryo group were analysed by t.l.c. for various metabolites which were subsequently identified by crystallization to constant specific activity with authentic steroids. All t.l.c. plates (Whatman cat. no. 4410222 ; polyester sheets coated with a $250 \mu \mathrm{m}$ layer of silica gel and a u.v. indicator at $254 \mathrm{~nm}$ ) containing the samples and authentic steroids were developed once in chloroform:ether (10:3, v/v) (Wiebe, 1982). Five steroid fractions were collected: namely, $5 \alpha$-pregnan-3,20-dione (or $5 \alpha$-dihydroprogesterone, $5 \alpha$-DHP), progesterone,

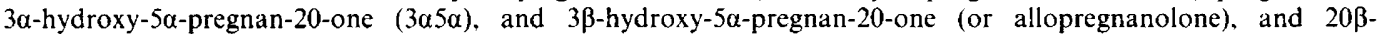

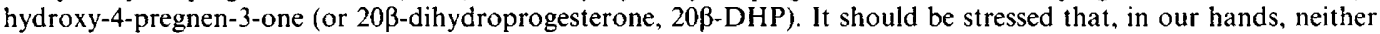
this solvent system nor Wiebe's complete two-dimensional solvent system (Wiebe, 1982) could adequately separate allopregnanolone from pregnenolone. An earlier study of progesterone metabolism by cultured Day -4 and -5 blastocysts (Wu, 1987) indicated that the appearance rate of allopregnanolone was about 3.5 times that of $3 \alpha 5 \alpha$ in Day-5 blastocyst cultures and $1 \cdot 5-4 \cdot 5$ times of that in Day-4 blastocyst cultures. The appearance rate of allopregnanolone in the present study was therefore very conservatively estimated (particularly during the second half of culture) to be twice that of $3 \alpha 5 \alpha$ at all time periods.

Identification of pregnenolone metabolites. As indicated above, only two Day-5 blastocyst cultures were used for the isolation and identification of metabolites at the end of culture. The selection of Day-5 cultures over the Day- 3 and -4 ones was based solely on the observation that Day- 5 embryos were metabolizing pregnenolone at a much faster rate than the younger ones, and so larger amounts of metabolites could be isolated for identification.

Three metabolites, namely, progesterone, $5 \alpha$-DHP and $3 \alpha 5 \alpha$, were isolated and identified by crystallization to constant specific activity from methanol, acetone:ethanol $(1: 1 \mathrm{v} / \mathrm{v})$, and methanol:water $(9: 1 \mathrm{v} / \mathrm{v})$, respectively. The crystallization procedure (Wu, 1987) was repeated for as many times as the sample allowed (3-7 times). All the crystal samples were dried, weighed and dissolved in the scintillation cocktail Opti-Fluor (Packard Instrument Co, Downers Grove, IL, USA) for counting the radioactivity in a Packard Tri-Carb liquid scintillation analyser at an efficiency of $47 \%$. The c.p.m./mg crystals was calculated for each sample. The values of the last two crystal samples which varied less than $3 \%$ were averaged and calculated as the percentage of the starting sample remaining as the authentic steroid.

Calculation of metabolite appearance rate. The appearance rate $(\mathrm{R})$ was calculated as follows:

$$
R=\frac{V\left(c \cdot p \cdot m_{\cdot 12}-c \cdot p \cdot m_{t_{11}}\right) p}{S \cdot C \cdot E \cdot B\left(t_{2}-t_{1}\right)}
$$

where $\mathrm{V}=$ the volume of medium in the culture, in $\mu \mathrm{l}$; c.p.m. $\mathrm{m}_{12}$ and c.p. $\mathrm{m}_{11}=$ the c.p.m. values of the metabolite fraction in $1 \mu \mathrm{l}$ medium at culture times of $t_{2}$ and $t_{1} h$, respectively; $p=$ purity of the metabolite fraction; $S=$ specific activity of $\left[{ }^{3} \mathrm{H}\right]$ pregnenolone, in d.p.m./fmol; $\mathrm{C}=$ the counting efficiency; $\mathrm{E}=\mathrm{t} . \mathrm{l} . \mathrm{c}$. recovery rate; and $\mathrm{B}=\mathrm{the}$ number of embryos in the culture.

In the control cultures, the appearance rates of various metabolites were calculated on the assumption that they contained as many embryos as did the experimental cultures. The control values served not only as the baselines for assessing the experimental data but also as indicators of experimental variations from sampling, t.l.c. and scintillation procedures.

Statistical analysis. The appearance rates of progesterone and its metabolites were compared (a) within culture groups (i.e. Day 3,4 or 5) between successive days of culture; and (b) between culture groups on the corresponding day in culture, by the $t$ test, with Bonferonni's adjustment for 5 comparisons (Miller, 1981).

\section{Results}

The t.l.c. analysis of daily samples from cultures of Day-3, -4 , and -5 embryos in $\left[{ }^{3} \mathrm{H}\right]$ pregnenolone medium indicated that the radioactivity in progesterone and its metabolite fractions (5a-DHP and $3 \alpha 5 \alpha$ ) continued to increase with time. Recrystallization experiments (Table 1) showed that the purity of progesterone, $5 \alpha$-DHP and $3 \alpha 5 \alpha$ was $72 \cdot 1,71 \cdot 7$ and $77 \cdot 5 \%$, respectively. These values were used to estimate the amount of various metabolites present in their respective fractions.

The appearance rates of progesterone, $5 \alpha$-DHP and $3 \alpha 5 \alpha$ are shown in Table 2. All three steroids were found in the $24-\mathrm{h}$ culture of Day-3 embryos. The appearance rates were generally increased with the age of embryos recovered and with the time in culture. The data were quite 
Table 1. Percentage purity of some metabolite fractions as determined by recrystallization to constant specific activity

\begin{tabular}{lccc}
\hline $\begin{array}{l}\text { Steroid } \\
\text { metabolites }\end{array}$ & Culture 1 & Culture 2 & Mean \\
\hline Progesterone & $61 \cdot 8$ & $82 \cdot 4$ & $72 \cdot 1$ \\
$5 \alpha$-DHP & $79 \cdot 3$ & $64 \cdot 1$ & $71 \cdot 7$ \\
$3 \alpha 5 \alpha$ & $76 \cdot 0$ & $79 \cdot 0$ & $77 \cdot 5$ \\
\hline
\end{tabular}

variable so many of the experimental values were statistically not significantly different from the controls, even though the means were different.

\section{The appearance rate of progesterone and its metabolites}

Figure 1 shows the combined amounts of progesterone, $5 \alpha$-DHP, $3 \alpha 5 \alpha$ and allopregnanolone formed by the Day-3, -4 , and -5 embryos cultured with $5 \mu \mathrm{M}$-pregnenolone. The results should be considered only as the 'gross progesterone formation rate' as there might well be other metabolites of progesterone in the medium that have yet to be identified.

When Day- 3 mouse embryos were cultured in medium with $5 \mu \mathrm{M}$-pregnenolone, progesterone and its metabolites were produced at the rate of $32 \pm 6$ (s.e.m.) fmol/h/embryo during the lst day. The rate rose by half the 2 nd day, then it increased 4 -fold $(P<0 \cdot 01)$ to $221 \pm 30 \mathrm{fmol} / \mathrm{h} / \mathrm{embryo}$ during the 3 rd day and a further $50 \%$ the 4 th day.

Table 2. Appearance rates $\left(10^{-3} \mathrm{fmol} / \mathrm{h} / \mathrm{embryo}\right)$ of (a) progesterone, (b) $5 \alpha$-dihydro progesterone ( $5 \alpha$ -

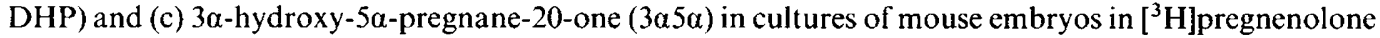

\begin{tabular}{|c|c|c|c|c|c|c|}
\hline \multirow{2}{*}{$\begin{array}{l}\text { Culture } \\
\text { group }\end{array}$} & & \multirow[b]{2}{*}{ No. } & \multicolumn{4}{|c|}{ Time in culture } \\
\hline & & & $0-22 \mathrm{~h}$ & $22-46 \mathrm{~h}$ & $46-70 \mathrm{~h}$ & $70-94 \mathrm{~h}$ \\
\hline \multicolumn{7}{|c|}{ (a) Progesterone } \\
\hline \multirow[t]{2}{*}{ Day 3} & Embryo & 4 & $2 \cdot 5 \pm 0 \cdot 5_{\mathrm{d} . \mathrm{b}}^{\mathrm{A}}$ & $2 \cdot 6 \pm 2 \cdot 2_{\mathrm{c}}^{\mathrm{B}}$ & $52 \pm 6^{\mathrm{A} \cdot \mathrm{B}}$ & $120 \pm 45$ \\
\hline & Control & 4 & $0.4 \pm 0.7$ & $0.9 \pm 0.4$ & $0 \cdot 6 \pm 0 \cdot 6$ & $0.5 \pm 0.2$ \\
\hline Day 4 & Embryo & 4 & $106 \pm 20_{a}$ & $65 \pm 32_{d}$ & $160 \pm 63$ & $18 \pm 28$ \\
\hline \multirow{2}{*}{ Day 5} & Embrvo & $\begin{array}{l}3 \\
3\end{array}$ & $\begin{array}{c}0.5 \pm 1.5 \\
437+83\end{array}$ & $\begin{array}{l}1.3 \pm 0.0 \\
726+15^{c}\end{array}$ & $\begin{array}{r}2 \cdot 4 \pm 3 \cdot 2 \\
178+48 c\end{array}$ & \\
\hline & Control & 2 & $\begin{array}{l}4.4 \pm 0.5 \\
0.4 \pm 0.2\end{array}$ & $0.4 \pm 0.2$ & $\begin{array}{l}1.4 \pm 48 \\
3 \cdot 4 \pm 3\end{array}$ & \\
\hline \multicolumn{7}{|c|}{ (b) $5 \alpha-\mathrm{DHP}$} \\
\hline \multirow[t]{2}{*}{ Day 3} & Embryo & 4 & $1.3 \pm 0.9^{\mathrm{A} . \mathrm{C}}$ & $6 \cdot 5 \pm 0 \cdot 8^{B . D}$ & $43 \pm 7_{\cdot}^{\text {A.B }}$ & $138 \pm 34^{C . D}$ \\
\hline & Control & 4 & $0.7 \pm 0.6^{\mathrm{a}}$ & $1 \cdot 4 \pm 1 \cdot 4^{\text {c.d }}$ & $2 \cdot 6 \pm 1.9$ & $1.0 \pm 0.7$ \\
\hline \multirow[t]{2}{*}{ Day 4} & Embryo & 4 & $20 \pm 6_{b}$ & $65 \pm 15_{c . c}$ & $121 \pm 32_{\mathrm{g}}$ & $418 \pm 265$ \\
\hline & Control & 3 & $2 \cdot 1 \pm 3 \cdot 3$ & $1.5 \pm 2.5$ & $0 \cdot 7 \pm 3.4$ & $0 \cdot 1 \pm 5 \cdot 1$ \\
\hline \multirow[t]{2}{*}{ Day 5} & Embryo & 3 & $306 \pm 3 I_{a, b}^{\mathrm{Fa}}$ & $1642 \pm 154_{d \alpha c}^{\mathrm{E}, G}$ & $849 \pm 46_{f, g}^{F G G}$ & \\
\hline & Control & 2 & $3 \cdot 2 \pm 2 \cdot 5$ & $28 \pm 34$ & $0.9 \pm 16.4$ & \\
\hline \multicolumn{7}{|l|}{ (c) $3 \alpha 5 \alpha$} \\
\hline \multirow[t]{2}{*}{ Day 3} & Embryo & 4 & $11 \pm 1 \cdot 5_{a}$ & $12 \pm 3 \cdot 6_{b, c}$ & $42 \pm 13$ & $32 \pm 10$ \\
\hline & Control & 4 & $1.0 \pm 0.8$ & $1.9 \pm 0.6$ & $1.2 \pm 0.6$ & $0.6 \pm 1.4$ \\
\hline \multirow[t]{2}{*}{ Day 4} & Embryo & 4 & $46 \pm 5_{a}^{A . B}$ & $96 \pm 11_{b, d}^{A}$ & $100 \pm 15$ & $126 \pm 11^{B}$ \\
\hline & Control & 3 & $7 \pm 4$ & $0.7 \pm 1.9$ & $11 \pm 6$ & $0.7 \pm 1 \cdot 1$ \\
\hline \multirow[t]{2}{*}{ Day 5} & Embryo & 3 & $77 \pm 20$ & $598 \pm 117$ & $748 \pm 239$ & \\
\hline & Control & 2 & $7 \pm 0.5$ & $5 \pm 3$ & $26 \pm 20$ & \\
\hline
\end{tabular}

Values (mean \pm s.e.m.) for each substance with the same superscript (within cultures) or subscript (between cultures) are significantly different: (a) A,C $P<0.005$; B $P<0.05$; a,b $P<0.05$; c, $P<0.01$; (b) A,E,F $P<0.01$; B,C,D,G $P<0.05$; a,b,d,e,f,g,h $P<0.01$; c $P<0.05$; (c) A $P<0.05$; B $P<0.01$; a,b,d $P<0.01$; c $P<0.05$. 


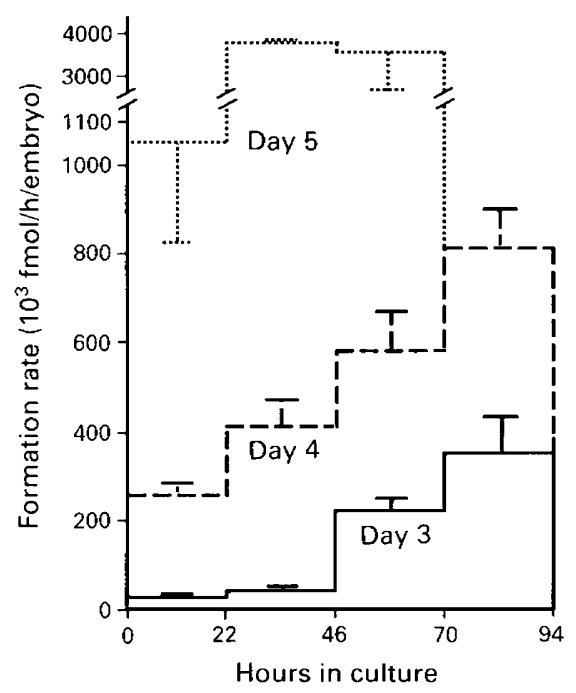

Fig. 1. Formation of progesterone and its metabolites (mean \pm s.e.m.) by mouse embryos cultured in pregnenolone medium. The vertical bar $=$ s.e.m.; $n=4,4$ and 3 cultures for Day-3, -4 , and -5 embryos, respectively.

The Day- 4 mouse embryos were much more active than the Day-3 ones and produced 8 times as much as did the latter $(264 \pm 26 \mathrm{fmol} / \mathrm{h} / \mathrm{embryo}, P<0.01)$ on the 1 st day. The rate was increased by $40-50 \%$ on each of the next 3 days.

The Day-5 embryos (implanting blastocysts) produced progesterone and its metabolites at the rate of $1051 \pm 218 \mathrm{fmol} / \mathrm{h} / \mathrm{embryo}$ on the first day, approximately 4 times that of Day- 4 embryos. The production rate increased by almost 4 -fold to $3808 \pm 96 \mathrm{fmol} / \mathrm{h} / \mathrm{embryo}$ on the $2 \mathrm{nd}$ day and decreased slightly on the 3 rd day.

\section{Discussion}

The present study has established beyond doubt that Day- 3 mouse embryos can convert pregnenolone into progesterone and that the latter is further metabolized into various metabolites. Although only 2 of the 4 known progesterone metabolites were isolated and identified, there was no reason to believe that the other two should not be present in the medium as the culture conditions were similar to those reported earlier (Wu, 1987). Furthermore, in cultures containing embryos, the amount of radioactivity remaining at the origin after t.l.c. increased with the culture time. This did not occur in the control culture. It is therefore highly likely that additional metabolites of pregnenolone and/or progesterone, more polar than those known ( $\mathrm{Wu}, 1987)$, might be generated by the mouse embryos, and the total amount of pregesterone and its metabolites produced by the embryos could be considerably higher than that presented.

It is difficult to explain why the present study has succeeded whilst others (Sherman \& Atienza, 1977; Antila et al., 1977) have failed in detecting the production of progesterone from pregnenolone by preimplantation mouse embryos. Certainly, there are many differences in the experimental details, e.g. the strain of mouse used, the number of embryos per unit volume of culture medium (i.e. embryo density), the specific activity and concentration of the radioactive substrate used, the time in culture, and the culture wares used, and all could have contributed to the outcome. In our experimental design, within the mouse strain used, we have tried to maximize the values of other 
factors so that a greater sensitivity in the detection of progesterone formation by the embryos can be achieved. Our familiarity with progesterone metabolites (Wu, 1987) also aided greatly in this study. For example, in Day-3 embryo cultures, progesterone present in the medium amounted to only $2.5 \times 10^{-3} \mathrm{fmol} / \mathrm{h} /$ embryo (Table 2) while its $3 \alpha 5 \alpha$ metabolite amounted to $11 \times 10^{-3} \mathrm{fmol} / \mathrm{h} / \mathrm{embryo}$ (Table 2). The amounts of progesterone metabolites could therefore exceed that of progesterone itself.

The results also show that the ability to convert pregnenolone into progesterone and related metabolites increased markedly with embryonic development. Thus, during the first $22 \mathrm{~h}$ in culture, the production rate of progesterone and its metabolites increased from $32 \mathrm{fmol} / \mathrm{h} / \mathrm{embryo}$ in Day-3 embryos (4-10-cells) to $264 \mathrm{fmol} / \mathrm{h} / \mathrm{embryo}$ in Day-4 embryos (early blastocysts) and to $1051 \mathrm{fmol} / \mathrm{h} /$ embryo in Day-5 embryos (late or implanting blastocysts), 7-fold and 3-fold increases, respectively. Once the embryos were placed in culture they appeared to take a longer time than in vivo to attain an elevated production rate of progesterone and its metabolites. For example, it took the Day-3 embryos 3 days in culture to reach the production rate exhibited by Day- 4 embryos on the first day in culture. Similarly, Day-4 embryos in culture for 4 days appeared to barely reach the rate shown by Day-5 embryos on the 1 st day of culture. These results suggest that the culture conditions as provided in the present study were far from ideal for the development of the enzymic activity for progesterone production and metabolism. This could be due to (1) the lack of some beneficial factors in the medium; (2) the presence of too much steroid in the medium; and (3) radiation damage from the tritiated steroids. Nevertheless, the culture conditions did allow the occurrence of hatching of blastocysts from the zona pellucida and attachment of the blastocysts to the glass tube.

The role of steroid synthetic and metabolic ability of the embryo in its own development remains to be elucidated. The embryonic steroid hormones might be needed for optimal development of the embryos in two ways: (1) the steroids might act directly on the embryonic cells themselves, although little is known about this, and (2) the embryonic steroids might act on the reproductive tract to provide a suitable milieu for the development of the embryo. During early pregnancy, both the oviduct and the uterus are under the influence of ovarian steroids. The imposition of embryonic steroids on the tract could cause some local effects. There are indications that preimplantation embryos can produce some local effects in the oviduct [e.g. elevated lactic acid level (Gupta et al., 1970), decreased thymidine incorporation (Freese et al., 1973), and modified embryo transport through the oviduct (Betteridge et al., 1976; Villalon et al., 1982)] and the uterus [e.g. increased capillary permeability (Psychoyos, 1973; Dickmann et al., 1977), increased uterine secretion (Nilsson, 1977; Aitken, 1977), and changes in oestrogen receptors (Martel \& Psychoyos, 1981; Puri \& Roy, 1981)]. It is also possible that the embryo's steroidogenic ability could be a mechanism to improve its chance of survival when the maternal source of steroid hormones is deficient for the optimal stimulation of the reproductive tract. However, it remains difficult to explain why the embryo should metabolize progesterone so soon after its formation. Perhaps too much progesterone is injurious to the embryo or progesterone metabolites might have some function in early pregnancy which is yet to be identified. In-vitro studies have shown that progesterone may be inhibitory to the development of the cleavage stage of mouse embryos at concentrations of $4 \mu \mathrm{g} / \mathrm{ml}$ (Whitten, 1957) and $8 \mu \mathrm{g} / \mathrm{ml}$ (Kirkpatrick, 1971) but stimulatory at 1 and $4 \mu \mathrm{g} / \mathrm{ml}$ (Roblero $\&$ lzquierdo, 1976). If progesterone concentration is related to the well being of the embryo, it is not difficult to visualize that the embryo might utilize its ability to metabolize progesterone as a means of regulating the concentration of progesterone inside itself and, probably, in its immediate surroundings. This could be a protective mechanism.

In summary, then, the preimplantation mouse embryo can make progesterone from pregnenolone and, probably, could regulate its progesterone level by means of its own enzymic ability.

This study was supported by an NICHD grant HD 21322. We thank NIDDK and the National Hormone and Pituitary Program, University of Maryland School of Medicine, for the gifts of PMSG and hCG; and Ms Marcia Bowles for typing the manuscript. 


\section{References}

Aitken, R. (1977) Changes in the protein content of mouse uterine fushings during normal pregnancy and delayed implantation, and after ovariectomy and oestradiol administration. J. Reprod. Fert. 50, 29-36.

Antila, E., Koskinen, J., Niemela, P. \& Saure, A. (1977) Steroid metabolism by mouse preimplantation embryos in vitro. Experientia 33, 1374-1375.

Betteridge, K.J., Flood, P.F. \& Mitchell, D. (1976) Possible role of the embryo in the control of oviductal transport in mares. In Ovum Transport and Fertility Regulation, pp. 381-389. Eds M. J. K. Harper, C. J. Pauerstein, C. E. Adams, E. M. Coutinho, H. B. Croxatto \& D. M. Patton. Scriptor, Copenhagen.

Dickmann, Z., Dey, S.K. \& Gupta, J.S. (1976) A new concept: control of early pregnancy by steroid hormones originating in the preimplantation embryo. Vitams Horm. 34, 215-242.

Dickmann, Z., Sengupta, J. \& Dey, S.K. (1977) Does 'blastocyst estrogen' initiate implantation? Science, $N Y 195,687-688$

Freese, U.E., Orman, S. \& Paulos, G. (1973) An autoradiographic investigation of epithelium-egg interaction in the mouse oviduct. Am. J. Obstet. Gynecol. 117, 364-370.

Grube, K.E., Gwazdauskas, F.C., Lineweaver, J.A. \& Vinson, W.E. (1978) Steroidogenic capabilities of the early mouse embryo. Steroids 32, 345-354.

Gupta, D.N., Karkun, J. \& Kar, A.B. (1970) Biochemical changes in different parts of the rabbit fallopian tube during passage of ova. Am. J. Obstet. Gynecol. 106, 833-837.

Kirkpatrick, J.R. (1971) Differential sensitivity of preimplantation mouse embryos in vitro to oestradiol and progesterone. J. Reprod. Fert. 27, 283-285.

Lopata, A., Johnston, I.W.H., Hoult, I.J. \& Speirs, A.L. (1980) Pregnancy following intrauterine implantation of an embryo obtained by in vitro fertilization of a preovulatory egg. Fert. Steril. 33, 117-120.
Martel, D. \& Psychoyos, A. (1981) Estrogen receptor in the nidatory sites of the rat endometrium. Science, NY 211, 1454-1455.

Miller, R.G., Jr (1981) Simultaneous Statistical Inference, 2nd edn. Springer-Verlag, New York.

Nilsson, O. (1977) Local secretory response by the mouse uterine epithelium to the presence of a blastocyst or blastocyst-like bead. Anat. Embryol. 150, 313-318.

Psychoyos, A. (1973) Hormonal control of ovoimplantation. Vitams Horm. 31, 201-256.

Puri, R.K. \& Roy, S.K. (1981) Estradiol receptors in embryonic and inter-embryonic segments of uterus of the rabbit during implantation. Ind. J. exp. Biol. 19, 26-28.

Roblero, L. \& Izquierdo, L. (1976) Effect of progesterone on the cleavage rate of mouse embryos in vitro. $J$. Reprod. Fert. 46, 475-476.

Sherman, M.I. \& Atienza, S.B. (1977) Production and metabolism of progesterone and androstenedione by cultured blastocysts. Biol. Reprod. 16, 190-199.

Villalon, M., Ortiz, M.E., Aguayo, C., Munoz, J. \& Croxatto, H.B. (1982) Differential transport of fertilized and unfertilized ova in the rat. Biol. Reprod. 26, $337-341$.

Whitten, W.K. (1957) The effect of progesterone on the development of mouse eggs in vitro. J. Endocr. 16, $80-85$.

Wiebe, J.P. (1982) Identification of a unique Sertoli cell steroid as $3 \alpha$-hydroxy-4-pregn-20-one ( $3 \alpha$-dihydroprogesterone: $3 \alpha$-DHP). Steroids 3, 259-278.

Wu, J.T. (1987) Metabolism of progesterone by preimplantation mouse blastocysts in culture. Biol. Reprod. 36, 549-556.

Wu, J.T. \& Lin, G.M. (1982) The presence of $17 \beta-$ hydroxysteroid hydrogenase activity in preimplantation rat and mouse blastocysts. J. exp. Zool. 220, $121-124$.

Received 27 February 1989 